\title{
Protocol Driven Approach to Optimize the Absorption of Physically Incompatible Drugs with Enteral
}

\section{Formulations}

\author{
William Ruspantini ${ }^{1}$, Movses Hovsepian ${ }^{1}$, Asha Bhalla ${ }^{2}$, Andrew $\mathrm{Chu}^{2}$, and Edwina Rich ${ }^{2}$ \\ 1. Department of Pharmacy, NYU Langone Medical Center, New York 10016, USA \\ 2. Department of Nutrition, NYU Langone Medical Center, New York 10016, USA
}

\begin{abstract}
The purpose of this study was to determine the feasibility of a protocol-driven approach to optimize the absorption of drugs physically incompatible with enteral nutrition in critical care patients on continuous tube feeds during 6 quarterly review periods. A hospital-based interdisciplinary protocol was approved in which physically incompatible drugs could be converted from the IV (intravenous) or oral formulation to an enteral formulation and administered during a "no tube-feed” interval. Eligible patients included those receiving continuous TF (tube feed formulas) and enterally administered medications for at least 2 days while receiving a physically incompatible target drug from the protocol. The primary outcome was the percentage of physically incompatible target drugs successfully converted to the appropriate enteral formulation and administration time. The secondary outcomes were the cost savings of all enteral target drugs converted during each quarter based on a 7 day review and the total cost savings of each target drug converted during the entire study. The primary outcome of successful drug conversions to the appropriate enteral administration was $100 \%$ for 5 of the 6 quarters and 81 percent for one quarter. The secondary outcome of cost savings of all enteral drug conversions averaged $\$ 975$ per quarter. Also, the cost savings of each of the enteral target drug conversions for the entire study was between $\$ 3,376$ for the most costly drug and $\$ 26$ for least costly drug. The study shows that an interdisciplinary protocol with "no tube-feed" intervals is a feasible and cost effective method to successfully optimize the absorption of physically incompatible drugs with enteral formulas during continuous tube feedings.
\end{abstract}

Key words: Protocol-driven, drugs, incompatible, enteral nutrition.

\section{Introduction}

Drug administration in hospitalized patients who have a functional GIT (gastrointestinal track) but are unable to swallow because of dysphasia, severe mucositis or head and neck surgery are often dependent on enteral feeding tubes for nutrition and drug administration. Such a route, although less invasive and more convenient for the patient and medical staff, can be quite challenging and fraught with many drug-related problems. Among these are the use of such drugs as anticholinergics, opiates and calcium channel blockers (Table 1) which can slow the GI motility and increase the residual volume of

\footnotetext{
Corresponding author: William Ruspantini, M.S., Pharm. D., CGP, research field: pharmacy. E-mail: william.ruspantini@nyumc.org.
}

gastric contents, cause nausea and vomiting, reduce the LES (esophageal sphincter pressure) tone and increase the risk of reflux and aspiration of gastric contents. Although these problems have been minimized with the placement of feeding tubes more distally (i.e. JT), there are other drug-related problems that can cause potential medication errors and adverse events if oral medications are inappropriately administered by a medical staff that is unaware of the different formulations and technical delivery designs of a drug.

For example, SR (sustained released) formulations designed for a prolonged and constant delivery of an active ingredient can be altered if they are inadvertently crushed for feeding tube administrations. This can result in erratic blood levels and adverse 
consequences, such as severe hypotension pursuant to the initial surge of an active ingredient released abruptly from the crushed SR formulation of an antihypertensive drug. This can be further complicated with rebound hypertension as the active ingredient is rapidly eliminated and becomes sub-therapeutic. Moreover, these fluctuating blood pressures can lead to more errors such as the inappropriate prescribing of higher or lower doses of the drug or the use of other medications to control blood pressure [1, 2].

Just as important, are drugs that are enteric coated to protect the active ingredient from the stomach's acidity and optimize its bioavailability or prevent irritating effects. Finally, the use of liquid formulations considered more convenient and less likely to clog feeding tubes can, in contrast, have unintended consequences because of the nature of the vehicle (Tables 2 and 3) [3]. For example, sorbitol containing liquids ( $>10-20 \mathrm{~g}$ sorbitol/day), such as carbamazepine and theophylline liquids or hyperosmolar liquid medications (i.e. $>100-400$ $\mathrm{mOsm} / \mathrm{kg}$ such as acetaminophen elixir, lithium citrate syrup and cimetidine solution) can cause nausea, bloating, cramping or diarrhea and result in inadequate drug absorption or delayed administrations. Such consequences lead to poor management of the intended condition and delay the delivery of nutrition and other medications [1, 4].

However, a more commonly overlooked problem is the physical incompatibility between some drugs and the enteral formulas. For example acidic liquids with a $\mathrm{pH}$ less than 4, such as brompheniramine elixir or lithium citrate syrup can denature proteins in the enteral formula while others, such as sucralfate, can form a bezoar precipitate that can clog the feeding tube [1-3] and delay further nutrition and drug administration as well as to their costly replacement.

Based on a study by Belknap and colleagues, as much as $74 \%$ of the hospital staff used at least 2 incorrect methods in administering drugs via the enteral route, contrary to the guidelines for their proper administration from ASPEN (American Society of Parenteral and Enteral Nutrition) and other evidence-based studies [3, 5-7]. Some of these issues included crushing the enteric coated or sustained released medications before administrations while others included failure to properly flush the feeding

Table 1 Medications that may aggravate GERD [3].

\begin{tabular}{ll}
\hline Anticholinergics & Theophylline \\
\hline Barbiturates & Biphosphonates \\
Dihydropyridine calcium channel blockers & Aspirin/NSAIDS \\
Dopamine & Iron \\
Estrogen & KCL \\
Nicotine & Progesterone \\
Nitrates & Opiates \\
\hline
\end{tabular}

Source: adapted from Pharmacotherapy Handbook, DiPiro, JT, 8th edition.

Table 2 Osmolality (mOsm/kg) of liquid medications [3].

\begin{tabular}{ll}
\hline Average osmolality (mOsm/kg) & \\
\hline Acetaminophen elixir, $65 \mathrm{mg} / \mathrm{mL}$ & 5,400 \\
Amantidine HCL soln $10 \mathrm{mg} / \mathrm{mL}$ & 3,900 \\
Cimetidine soln $60 \mathrm{mg} / \mathrm{mL}$ & 5,550 \\
Co-trimazole suspension & 2,200 \\
Diphenoxylate/atropine suspension & 8,800 \\
Lithium citrate syrup $1.6 \mathrm{mEq} / \mathrm{mL}$ & 6,850 \\
\hline
\end{tabular}

Table 3 Sorbitol content of liquid medications [3].

\begin{tabular}{lllll}
\hline Liquid medication & Strength (per mL) & \multicolumn{1}{l}{ Sorbitol (\% w/v) } & Avg. daily adult dose & Sorbitol daily equiv. dose (g/day) \\
\hline Acetaminophen & $32 \mathrm{mg}$ & $<20$ & $1.3-3.9$ & $<8-24$ \\
Amantidine HCL & $10 \mathrm{mg}$ & 72 & $200 \mathrm{mg}$ & 14.3 \\
Carbamazepine (Tegretol) & $20 \mathrm{mg}$ & 17 & $400-1,200$ & $3.4-10.2$ \\
Ibuprofen & $20 \mathrm{mg}$ & 10 & $1.2-3.2$ & $6-16$ \\
Lithium citrate & $1.6 \mathrm{mEq}(8 \mathrm{mEq}=300 \mathrm{mg})$ & 54 & $900-1,800 \mathrm{mg}$ & $8.1-16.2$ \\
KCL & $1.33 \mathrm{mEq}$ & 17.5 & $16-100 \mathrm{mEq}$ & $2.1-13.1$ \\
Theophylline (Roxane) & $5.33 \mathrm{mg}$ & 45.5 & $150-600 \mathrm{mg}$ & $12.8-51.2$ \\
Valproate Na (Depakene) & $50 \mathrm{mg}$ & 15 & $1-4 \mathrm{~g}$ & $3-12$ \\
\hline
\end{tabular}


Table 4 Enteral-Drug administration/interaction guidelines [2, 4].

\section{Procedure:}

(1) N/G medications should only be given if there is no evidence of more than $250 \mathrm{~mL}$ of residual volume from bolus or continuous feeds (large volume residuals impair drug absorption).

(2) N/G medications should be avoided in patients with diarrhea, decompensated heart failure or ileus.

(3) Flush crushed medications with*20-30 mL sterile water before and after administration.

(4) Shaked suspensions and oral solutions should be mixed with $30 \mathrm{~mL}$ sterile water if viscous and flushed with $30 \mathrm{~mL}$ sterile water before and after administration.

(5) Tube feeds to be held between 8 am to 12 noon $+/-8$ pm to 12 midnight.

(6) Sinemet administration exception: Hold tube feeds $1 \mathrm{hr}$ before and after each Sinemet administration.

(7) Sorbitol Containing Medications-Monitor for nausea, bloating and diarrhea with following:

Carbamazepine suspension, acetaminophen elixir, kayexalate suspension, guaifenesin syrup , theophylline solution, valproate, syrup-monitor for nausea, bloating and diarrhea.

\begin{tabular}{|c|c|}
\hline Medications administered with & Enteral tubes to be flushed as indicated below \\
\hline Warfarin & $\begin{array}{l}\text { Administer at 9PM. Avoid enteral formulas }>80 \mathrm{mcg} / 1000 \mathrm{Kcal} \text { of vitamin K. NYU enteral } \\
\text { formulas contain }<80 \mathrm{mcg} / 1000 \mathrm{Kcal} \text {. Monitor INR during T.F.'s and when it is discontinued. } \\
\text { Food decrease rate, not extent of absorption. Soy protein caseinate in tube feeds may bind } \\
\text { warfarin. }\end{array}$ \\
\hline Moxifloxacin, Levofloxacin & Administer at 10 am according to guidelines \#3 and \#5 above. \\
\hline Ciprofloxacin & Administer at 10 am according to guidelines $\# 3$ and $\# 5$ above. \\
\hline Phenytoin & Administer 10 am and 10 pm according to guidelines \#4 and \#5 above. \\
\hline Carbamzepine & $\begin{array}{l}\text { Administer at } 10 \mathrm{am} \text { and } 10 \mathrm{pm} \text { as crush tablets or administer carbamazepine suspension } \\
\text { according to guidelines } \# 3, \# 4, \# 5 \text { and } \# 7 \text { above. }\end{array}$ \\
\hline Sinemet & $\begin{array}{l}\text { Change to oral disintegrating tablets (Parcopa) or administer crush regular release tablets } \\
\text { according to guidelines \#6 above. }\end{array}$ \\
\hline Ampicillin, penicillin & Follow direction on ICIS prompt screens for antibiotic substitution in tube feed patients. \\
\hline Cephalexin & Follow direction on ICIS prompt screens for antibiotic substitution in tube feed patients. \\
\hline Tetracycline & Follow direction on ICIS prompt screens for antibiotic substitution in tube feed patients. \\
\hline Isoniazid/Rifampin & Administer at 10 am according to guideline \#3 and \#5 above. \\
\hline Antacids & $\begin{array}{l}\text { Stop TF for one hour during feeds, administer dose and flush with } 30 \mathrm{ml} \text { sterile water before } \\
\text { restarting. Should NOT be administered via PEJ (intestinal feedings). Aluminum from antacids } \\
\text { forms obstructing proteins. }\end{array}$ \\
\hline Voriconazole & $\begin{array}{l}\text { Administer at } 10 \text { am according to guidelines \#3 and \#5 above. If patient is receiving enteral feeds } \\
\text { with Glucerna or Pulmocare, call nutrition (\#2115) or pharmacy (x38080). }\end{array}$ \\
\hline Digoxin & $\begin{array}{l}\text { Administer at } 10 \text { am according to guidelines } \# 3 \text { and } \# 5 \text {. If patient is receiving enteral feeds with } \\
\text { Gevity 1.5, call nutrition (\#2115) or pharmacy (x38080). }\end{array}$ \\
\hline Synthroid & Adminster 10 am according to guidelines \#3 and \#5 above. \\
\hline $\begin{array}{l}\text { Oral biphosphonates } \\
\text { (i.e.risedronate) }\end{array}$ & $\begin{array}{l}\text { Administer crushed tablet at } 10 \mathrm{am} \text { with } 180 \mathrm{~mL} \text { of water } 30 \text { minutes before any other } \\
\text { medications. Patient to sit-up for } 30 \text { minutes and resume T.F.'s } 12 \text { noon. }\end{array}$ \\
\hline $\begin{array}{l}\text { HIV medications: } \\
\text { Didanosine, indinavir, amprenavir }\end{array}$ & Administer at 10AM and / or 10PM according to guidelines \#3 and \#5 above. \\
\hline
\end{tabular}

*Means $20 \mathrm{ml}$ used for fluid restricted patients.

tube. However, to apply such guidelines successfully would require an interdisciplinary and coordinated effort by nursing, pharmacy, nutrition and the prescriber.

A study done by Van den Bemt and colleaques investigated such an integrated approach to improve the bioavailability and reduce the error and complication rate (ielogged feeding tubes) of orally administered drugs in tube fed patients in 2 Dutch hospitals [8]. The program which included administration guidelines, nursing education and pharmacy consultation was implemented through an interdisciplinary project team at each hospital. In addition, contraindications to enteral administration of specific drugs were noted in the hospital computer system, caution labels, such as "do not crush" were placed on all SR and EC drugs dispensed and nursing instructions, such as timing the administration of incompatible drugs and the flushing of feeding tubes were implemented as well. The results of the study comparing data before and after the implementation of 
the protocol demonstrated over a 2-month period a trend towards less tube obstructions in hospital 1 and a significant decrease in drug administration errors in hospital 2.

Based on some of these past initiatives and promising results, we piloted a hospital-based interdisciplinary protocol to convert critical care patients on continuous tube feeds from either the IV drug or oral formulations of physically incompatible drugs with the nutrition formulas to an appropriate enterally administered drug during a "no-tube feed" interval.

\section{Materials and Methods}

Based on the success of this past approach, a major academic institution with the approval of the pharmacy and therapeutic committee formulated in cooperation with the institution's nutrition and pharmacy subcommittee an enteral drug administration and interaction protocol (Table 4) implemented through an interdisciplinary team of nurses, prescribers, nutritionists and a dedicated clinical pharmacist. The protocol was piloted in the ICU for 6 consecutive quarters after a 6-month orientation outlining the responsibilities of each service member during a trial period. The study included only tube fed patients receiving continuous enteral nutrition and medications via the enteral route for a minimum of 2 days. The protocol target drugs for review and intervention included both the IV and oral formulations on page 1 of the guidelines in Table 4 (i.e. fluoroquinolones, phenytoin, levothyroxine, etc.). According to the protocol, the target drugs were administered either once or twice daily: at 10 am or 10 am and $10 \mathrm{pm}$. The tube feeds were withheld between 8 am and 12 noon for 10 am administered target drugs and between 8 am and 12 noon and 8 pm and 12 midnight for target drugs administered at $10 \mathrm{am}$ and $10 \mathrm{pm}$, respectively. The protocol instructions included flushing procedures and other precautions outlined in the procedure section (Table 4).
The interdisciplinary protocol required the pharmacist to filter all ICU patients from the hospital computer system every day and identify all tube fed patients receiving this route for a minimum of 2 days (48 hours). The current medication profiles of the tube fed patients were reviewed for the IV and/or oral formulations of the target drugs. As long as the patients tolerated their tube feeds and enteral-administered medications, the IV formulations or the inappropriately timed oral administration of the targeted drugs was considered for conversion as per protocol.

After a brief discussion and approval by the prescribing physician, all eligible targeted drugs for conversion were changed in the hospital computer system to the enteral formulation and administered at $10 \mathrm{am}$ for once daily administrations and at $10 \mathrm{am}$ and $10 \mathrm{pm}$ for twice daily administrations with labeled instructions to the nurse (that also appeared in the nurse's electronic medication administration record) to withhold the tube feeds between 8 am and 12 noon for once daily administrations and, again, at $8 \mathrm{pm}$ and 12 midnight, for twice daily administrations ( "no tube feed intervals").

The covering nutritionist was then contacted to increase the TF rate to compensate for the "no tube feed intervals" to be sure the patient received the entire 24 hours nutrition order (i.e. if the original continuous TF rate was $40 \mathrm{~mL} / \mathrm{h} \times 24 \mathrm{~h}$ and there was a "no tube feed interval" only between 8 am and 12 noon or $4 \mathrm{hrs}$, the rate was adjusted to $60 \mathrm{~mL} / \mathrm{h} \times 16 \mathrm{~h}$ to ensure the $24 \mathrm{hr}$ feed of $960 \mathrm{~mL}$ was maintained).

The final step included contacting the covering nurse to inform her of the new order modified for the appropriately timed enteral administration of the targeted drug (i.e. $10 \mathrm{am}$ or $10 \mathrm{am}$ and $10 \mathrm{pm}$ depending on the schedule of the target drug) and the withholding of the tube feeds during the "no tube feed interval". The intervention was followed up with each shift nurse covering the patient so they were aware of the change as well. 
The primary endpoint was the percentage of eligible targeted drugs converted successfully as per protocol for a minimum of 7 days for each of 6 quarters. This endpoint served to measure the interdisciplinary staff compliance and feasibility of such an approach. The secondary endpoints were the "total target drug cost savings per quarter" and the "cumulative cost savings for each target drug during the entire 6 quarter review”.

\section{Results}

As Fig. 1 illustrates, there were between 171 and 394 patients in the ICU area per quarter and between $17 \%$ and $38 \%$ (average 26\%) of these received continuous tube feedings for a minimum of 48 hours. Among the tube fed patients, there were between 7 and 19 target drugs (average 12) per quarter (Fig. 2) that were eligible for conversion as per protocol to the appropriate enteral administration times of 10 am plus or minus $10 \mathrm{pm}$. As Fig. 3 illustrates, the primary endpoint of target drug conversions (a measure of staff compliance) was realized at $100 \%$ per quarter for 5 of the 6 quarters with a minimum patient follow-up of 7 consecutive days after the intervention. The lower compliance rate of $81 \%$ in the 6th quarter was due to patients that were either discharged from the ICU unit or deceased before implementing the intervention or the targeted drug was discontinued at any time within 7 days.

As Fig. 4 illustrates, the secondary endpoint of cost savings per quarter was between $\$ 771$ and $\$ 1,500$ and dependent on the number and cost of the specific target drugs converted. Drugs that were costly, such as IV levothyroxine or voriconazole dominated the overall cost savings for those quarters which may have had fewer conversions compared to those quarters with many more conversions but with much less expensive target drugs (i.e. phenytoin or digoxin). However, the average cost savings/quarter was $\$ 975$ which should help balance the inequitable cost savings for different quarterly periods.

The other secondary endpoint of cumulative cost saving per drug for the entire 6 quarter study period was quite variable. As illustrated in Fig. 5, 59\% of all target drug conversions was for the most costly drug, IV levothyroxine, which factored greatly into its cumulative cost savings for the entire study. This was followed by conversions of IV fluoroquinolones and voriconazole that were also significant in number and

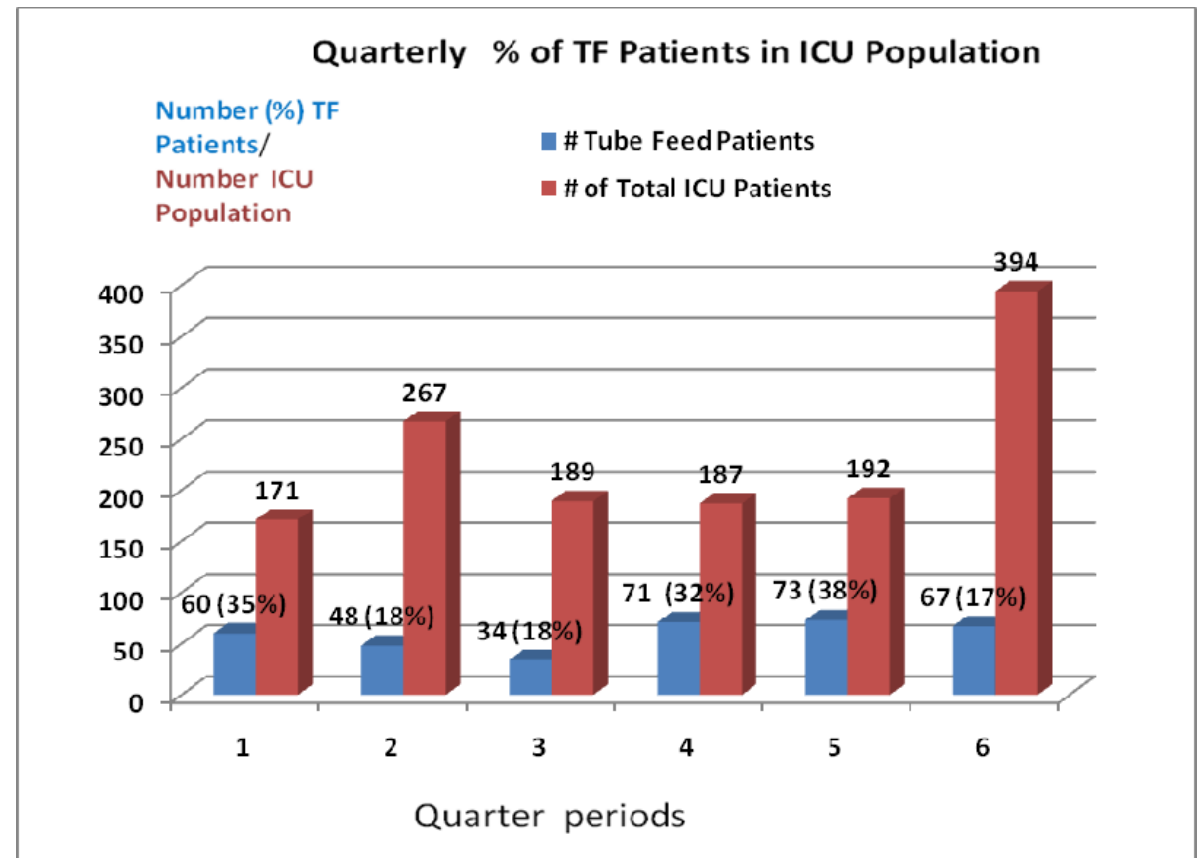

Fig. 1 Quarterly percentage of TF patients in ICU population. 


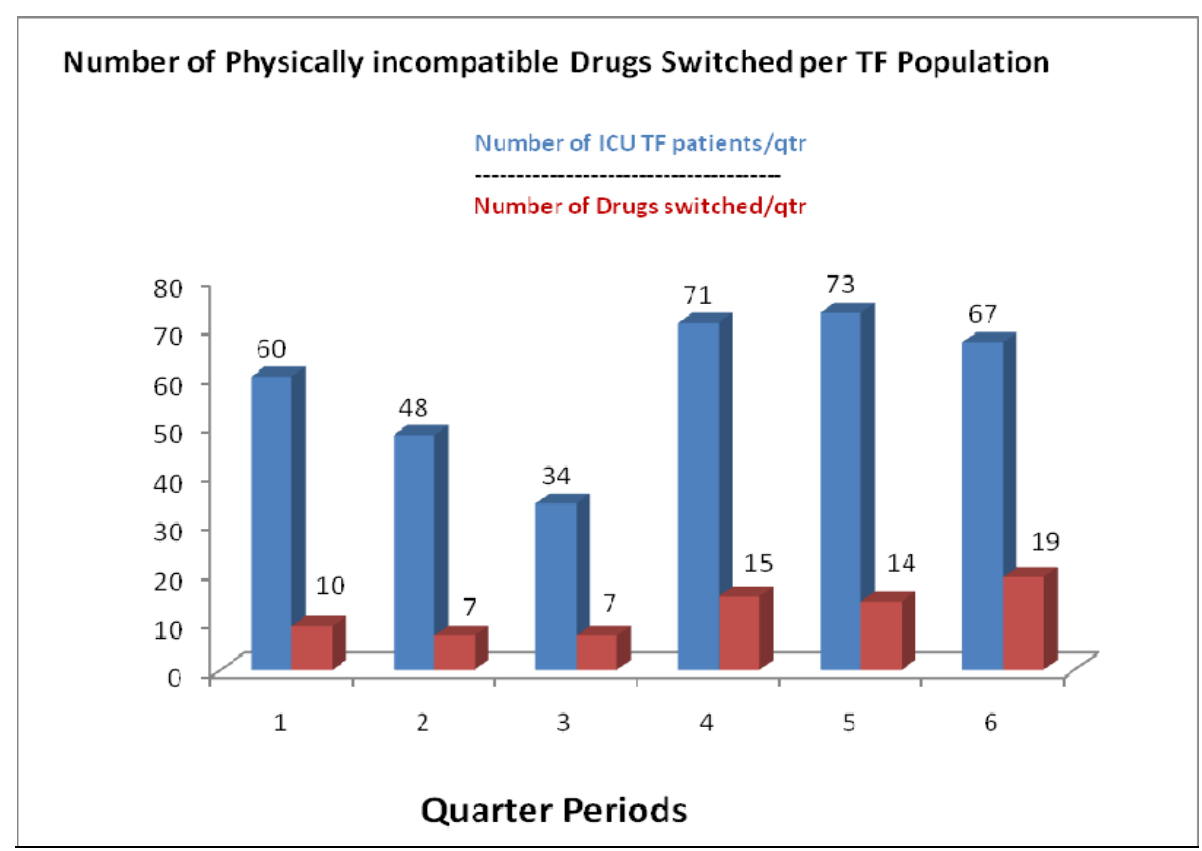

Fig. 2 Number of physically incompatible drugs switched in TF patients/quarter.

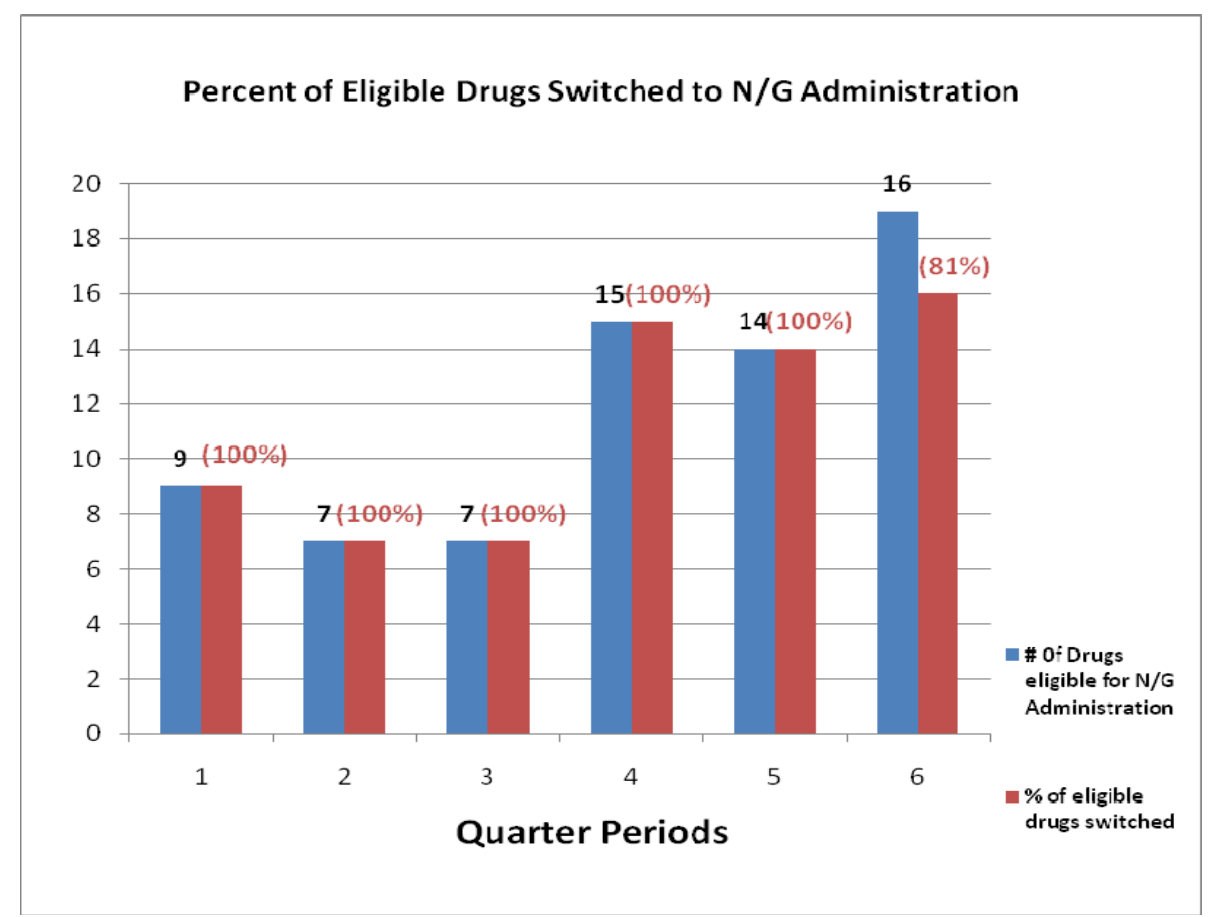

Fig. 3 Percentage of target drugs switched to appropriate enteral administration.

cost savings. As the graph illustrates, the relative number of conversions and the cumulative cost savings for the 6 quarter study period were highest for the more expensive drugs of levothyroxine (59\% of total drug conversions at a cost savings of \$3,376) and voriconazole (10\% of total drug conversions at a cost savings of $\$ 1,512)$ and lowest for phenytoin (4\% of total drug conversions at a cost savings of \$26).

\section{Discussion}

The enteral route has become increasingly used in the administration of nutrition and medications in hospitalized patients. Based on the lack of understanding and awareness of drug incompatibilities 


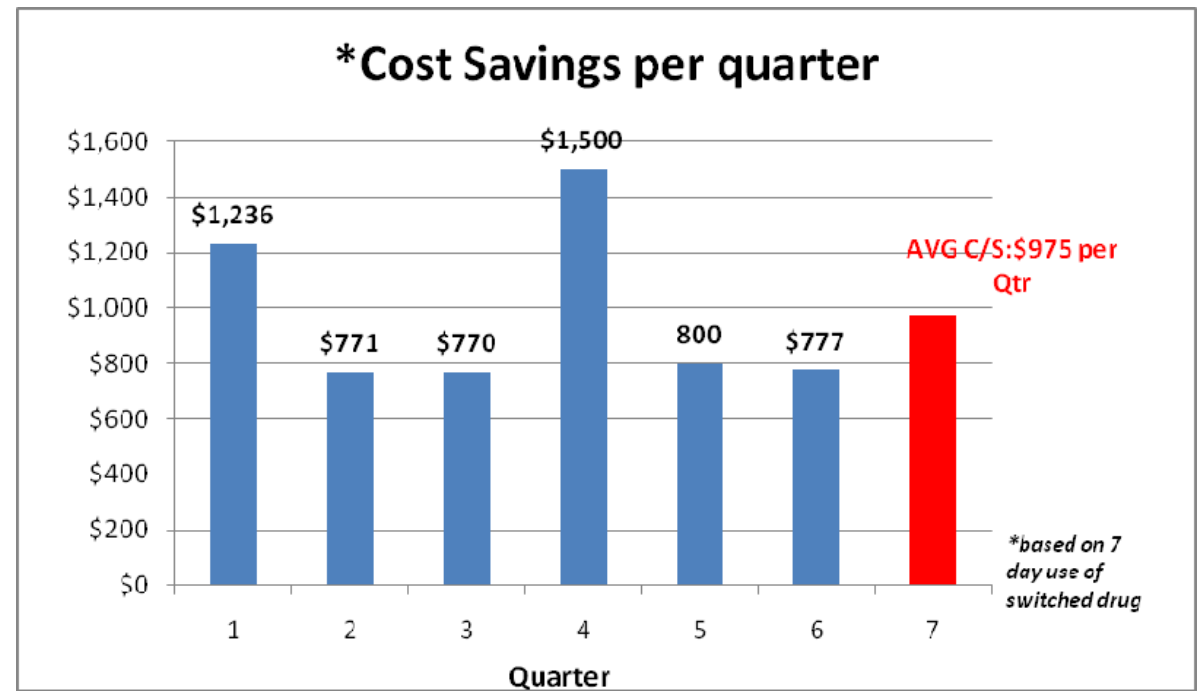

Fig. 4 Cost savings of all target drugs per quarter 1.

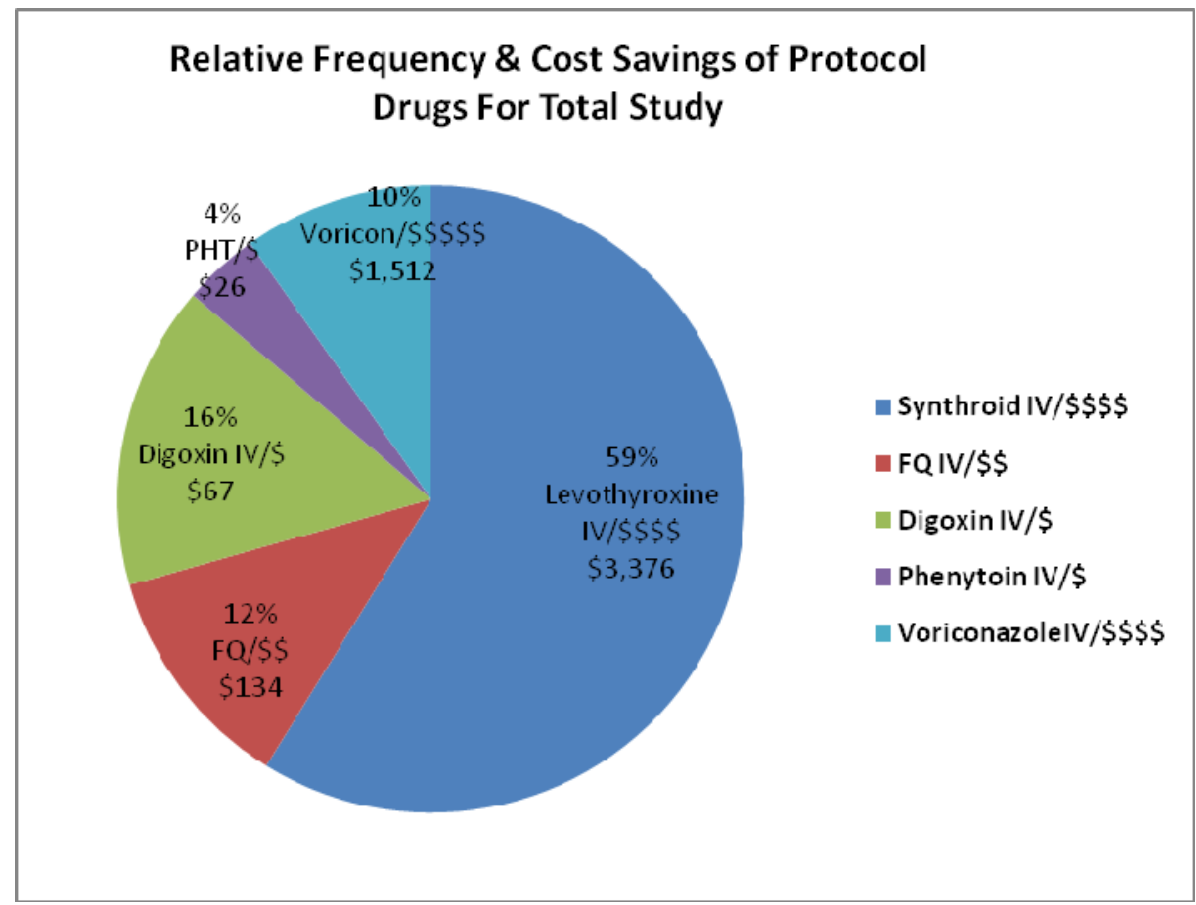

Fig. 5 Percentage of use and total cost savings per target drug in study.

and the misuse of sustained, enteric coated or hyperosmolar formulations in tube fed patients, there is an overdue need to identify and prevent these problems in practice. Although many institutions have COPE (computer order entry systems) that provide real time data on patient's demographics, medication profile, labs and nutritional status, along with tools to alert one to drug overdoses or drug-drug interactions, they may not have the logic to integrate data between a patient's use of enteral formulas with potentially incompatible drugs or dosage formulations. A protocol driven approach, such as the one used in this pilot study, might be a viable alternative to identify these problems. Although this requires staff education, training and cooperation and a dedicated pharmacist to be constantly vigilant and interactive, there may still be challenges with staff and resources to implement a more hospital-wide program. 
Among the limitations of this study was the lack of a 24/7 interdisciplinary approach since the review and guidelines were implemented between 8 am and $6 \mathrm{pm}$, Monday to Friday. It's possible there may have been missed opportunities during off hours to convert one or more doses of a target drug that would have resulted in different quarterly endpoint measures. Also patients receiving targeted drugs with less than a seven day ICU stay were excluded, although they may have continued the targeted drugs once they were transferred to another unit that was not included in this study but otherwise eligible for such a conversion.

Another limitation was the lack of clinical endpoints. Morbidity, mortality, length of therapy or ICU stays as well as the number of clogged tubes before and after the pilot program were not assessed to determine whether there would have been a significantly more favorable impact in these measures and cost savings.

Such endpoints as well as the impact of other drug-associated events ( i.e. ADRS, abnormal drug levels) with tube fed patients should be reviewed in future studies with more diverse patient populations to fully assess all the benefits from an interdisciplinary protocol-driven approach.

\section{Conclusion}

Use of an interdisciplinary protocol with 4 hour "no tube-feed intervals" is a feasible method to successfully convert unnecessary intravenous or oral formulations of drugs otherwise incompatible with enteral formulas to an appropriately timed enteral administration. Such an interdisciplinary protocol can improve patient care by assuring optimal bioavailability and therapeutic efficacy as well as cost savings for drugs commonly used in practice. Additionally, there may be other benefits that are more indirect but cost-effective as well. Among these may be the potential reduction in the length of therapy of nutritionally incompatible drugs and the reduced incidence of clogged tubes that would otherwise result in costly delays in therapy and tube replacements.

\section{References}

[1] Shahram, E., Hadi, M., Ata, M., Simin, M., and Parina, A. 2012. "Errors of Oral Medication Administration in a Patient with Enteral Feeding Tube.” J. Res. Pharm. Pract. 1 (1): 37-40.

[2] Joseph B. 2009. "Drug Administration through an Enteral Feeding Tube.” Am. J. Nurs. 109 (10): 34-42.

[3] Beckwith, M. C., Feddema, S. S., Barton, R. G., and Graves, C. 2004. “A Guide to Drug Therapy in Patients with Enteral Feeding Tubes: Dosage Form Selection and Administration Methods.” Hosp. Pharm. 39: 225-37.

[4] White, R., Bradnam, P., and Shaw Phillips, M. 2007. "Handbook of Drug Administration via Enteral Feeding Tubes.” Am. J. Pharm. Educ. 71 (5): 99.

[5] Belknap, D. C., Seifert, C. F. and Petermann M. 1997. "Administration of Medications through Enteral Feeding Catheters.” Am. J. Crit. Care 6 (5): 382-92.

[6] Bankhead, R., Boullata, J., Brantley, S., Corkins, M., Guenter, P., Krenitsky, J., Lyman, B., Metheny, N. A., Mueller, C., Robbins, S., and Wessel, J. 2009. "Medication Administration. In: A.S.P.E.N. Enteral Nutrition Practice Recommendations.” JPEN J. Parenter Enteral Nutr. 33 (2):158-62.

[7] Williams, N. T. 2008. "Medication Administration through Enteral Feeding Tubes.” Am. J. Health Syst. Pharm. 65 (24): 2347-57.

[8] van den Bemt, P. M., Cusell, M. B., Overbeeke, P. W., Trommelen, M., van Dooren, D., Ophorst, W. R., and Egberts, A. C. 2006. "Quality Improvement of Oral Medication Administration in Patients with Enteral Feeding Tubes.”_Qual. Saf. Health Care 15 (1): 44-7. 